\title{
Materials and Methods of Biosensor Interfaces With Stability
}

\author{
Min Song ${ }^{1}$, Xiaogang Lin ${ }^{1 *}$, Zhijia Peng ${ }^{1}$, Shibin $\mathrm{Xu}^{1}$, Lifeng $\mathrm{Jin}^{1}$, Xiaodong Zheng ${ }^{2 *}$ and \\ Haoyue Luo $^{1}$ \\ ${ }^{1}$ Key Laboratory of Optoelectronic Technology and Systems of Ministry of Education of China, Chongqing University, Chongqing, \\ China, ${ }^{2}$ Chongqing University Cancer Hospital, Chongqing University, Chongqing, China
}

Biosensors can convert the concentration of biological analytes into an electrical signal or other signals for detection. They are widely used in medical diagnostics, food safety, process control, and environmental monitoring fields. In recent years, new schemes of stable biosensor interfaces have attracted much attention. Interface design is a vital part of biosensor development, since its stability can be directly related to the quality of sensing performance such as sensitivity, stability, and linearity. This review summarized the latest methods and materials used to construct stable biosensor interfaces and pointed some future perspectives and challenges of them. From the literature, we found that

OPEN ACCESS

Edited by:

P. Davide Cozzoli,

University of Salento, Italy

Reviewed by:

Amay J. Bandodkar, Northwestern University,

United States

María Pedrero,

Complutense University of Madrid,

Spain

${ }^{*}$ Correspondence: Xiaogang Lin

xglin@cqu.edu.cn

Xiaodong Zheng

zxd0052005@163.com

Specialty section:

This article was submitted to Colloidal Materials and Interfaces,

a section of the journal

Frontiers in Materials

Received: 04 August 2020 Accepted: 20 November 2020

Published: 15 January 2021

Citation:

Song $M$, Lin X, Peng Z, XU S, Jin L, Zheng $X$ and Luo $H$ (2021) Materials and Methods of Biosensor Interfaces

With Stability.

Front. Mater. 7:583739.

doi: $10.3389 /$ /fmats.2020.583739 nanomaterials, polymers, and their composites such as chitosan, cellulose, and conducting polymers are the most common materials used in the biosensor interface design. Apart from materials, there are increasing developments in monolayer membrane techniques, three-dimensional constructions, and other interface techniques. This review is a study of the latest progress in biosensor interface stability solutions, which may provide some references and innovative directions of biosensor interface design for researchers in biosensor fields and encourage people to further explore new materials and methods.

Keywords: biosensor interfaces, materials, structures, preparation methods, stability

\section{INTRODUCTION}

\section{Biosensor}

A biosensor is a device that can transform chemical information coming from the concentration of biomolecules into useful analysis signals (Thévenot et al., 2001). It is widely used in the fields of medical diagnostics, food safety, process control, environmental monitoring, and so on (Kowalczyk, 2020). In the past 40 years, research on biosensors has made considerable progress, which benefits from the achievements of nanoscience, electronics, biotechnology, and silicon technology (Schuster, 2018). Furthermore, with the development of artificial intelligence (AI) and big data, intelligent biosensor has become a new hot research field. Biosensors play an increasingly important role in daily life and scientific research.

Biosensors basically contain the following four constructions: sensing elements (or receptors), which are used to specifically bind to the analyte to be tested; an interface, which provides a working environment for biosensor elements (Schmidt and Montemagno, 2004); a transducer, which transforms the physical or chemical information produced from the interaction between the sensing elements and the analyte into electrical signals (Turner, 2015); a series of electronic equipment including signal amplification, signal processing, and interface circuit for data analysis and processing (Cavalcanti et al., 2008). The structure of a biosensor is shown in Figure 1. 


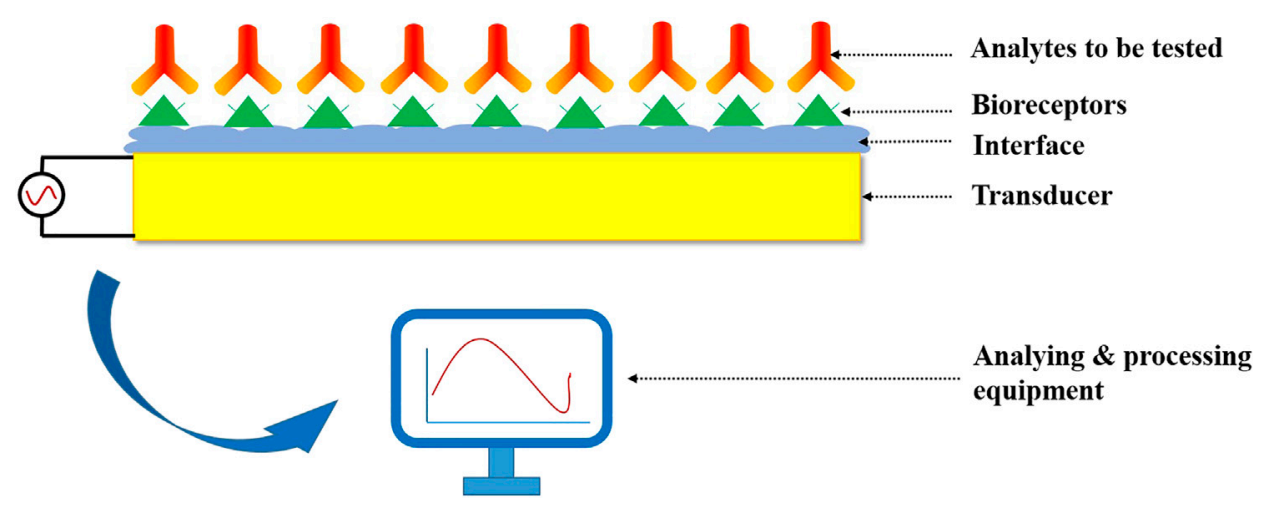

FIGURE 1 | The structure of a biosensor.

Generally, biosensors can be classified on the basis of three schemes. According to the different receptor types, biosensors can be divided into biocatalytic biosensors (i.e., enzyme), immunological biosensors (i.e., antibody), and nucleic acid biosensors (i.e., DNA). According to the physics of the transduction process, it involves electrochemical biosensor, optical biosensor, piezoelectric biosensor, and thermal or calorimetric biosensor. According to the application fields, it covers more types such as medical and clinical biosensors, environmental biosensors, and wearable biosensors. Moreover, commercial biosensors can be divided into two categories depending on whether they are laboratorial or portable (Nicu and Leichle, 2008). However, all of these biosensors need a stable interface to work properly and to have better performance in sensing.

\section{Interface of Biosensor}

How to combine the bionic system with silicon technology to form a functional interface is one of the most challenging work in biosensor design (Schmidt and Montemagno, 2004). A crucial step involved in it is the immobilization of biomolecules at the sensor surface. However, since biomolecules are highly susceptible to their environment and could denature rapidly due to changes in their surroundings, biomolecules cannot be directly immobilized on the surface of electrodes or other inorganic solid. In order to keep the biological activity of molecules, which is necessary for normal function of sensors, there should be a biocompatible intermedium layer between the sensing elements (or receptors) and the surface of electrodes (Matharu et al., 2012; Schuster, 2018). The layer is called a biosensor interface here. The stability of interface will affect the interaction of analytes on it and then affect the sensing performance of biosensor. Therefore, it is vital to design a stable interface in biosensor applications. This review tries to make a simple summary of the latest materials and structures in biosensor interface design and hopes to provide some references and innovation directions for biosensor researchers.

\section{Scope and Organization of the Review}

In this review, taking the stability of biosensor interfaces as the main line, some new materials and structures as well as preparation methods used in biosensor interface design in recent years are discussed. This review is organized as follows: firstly, Materials of Biosensor Interfaces with Stability introduces the materials commonly used in biosensor interfaces. Then, Structures and Preparation Methods of Stable Biosensor Interfaces summarizes the recent development in the interface structure of biosensors and gives some relevant examples. Finally, Conclusion makes a conclusion of these studies and gives the potential prospect as well as challenges in the future.

\section{MATERIALS OF BIOSENSOR INTERFACES WITH STABILITY}

\section{Nanomaterials} Metallic Nanomaterials

In recent years, the biosensor interface has made great improvement due to the development of nanomaterials. Nanomaterials can be used to improve the adsorption capacity of molecules, signal response speed, and the stability of the modified electrode because of their special physics and chemistry properties (Qian et al., 2014). There have been various nanomaterials and structures applied to biosensor interfaces such as nanospheres (Zhu et al., 2012), nanoporous structures (Matharu et al., 2017), nanotubes (Isaac et al., 2017), and nanowires (Hernández et al., 2016).

Gold nanoparticles (AuNPs) have the advantages of good microenvironment, large specific surface area, good biocompatibility, and high conductivity between the biomolecule and electrode surface, which makes them the ideal immobilization material for the fabrication of biosensor interfaces (Shi and Ma, 2011). Sun et al. developed a novel immunobiosensor based on glassy carbon (GC) electrode interface modified with AuNPs for carcinoembryonic antigen (CEA) detection. Experiment results showed just $4 \%$ current 
increase of the sensor within one month compared with the other two methods of $8 \%$ and $14 \%$, respectively (Sun and Ma, 2012), which indicated great stability of the AuNP-based biosensor. It is reported that some nanoparticles based on metal oxides have been successfully applied into biosensors. Thiagarajan et al. synthesized $\mathrm{ZnO}$ nanoparticles as the biosensor interface to immobilize the choline oxidase. $\mathrm{ZnO}$ has good catalytic property and biocompatibility, high surface-area-to-volume ratio, and high isoelectric point (IEP) of 9.5, which is helpful to immobilization of ChOx (IEP = 4.1) (Zhao et al., 2013). They evaluated the sensor performance in the detection of choline from breast cancer cells by using the hydrothermal method. The results showed a higher stability compared with the biosensors using other interfaces (Thiagarajan et al., 2016). Parlak et al. reported a nanoparticle-structured $\mathrm{MoS}_{2}$ nanosheet as the semiconductor interface of electrochemical glucose biosensors. The welldispersed $\mathrm{Au}$ nanoparticles were assembled by exploiting the affinity between the coordinated structures of $\mathrm{MoS}_{2}$ and $\mathrm{Au}$ nanoparticles. The results proved that it enhanced both electrocatalytic reactions and electrochemical properties (Parlak et al., 2017).

There are several other effects of nanomaterials applied in biosensor interfaces. For example, nanomaterials can act as mimic enzyme to replace bioenzyme to improve stability (Zhang and Wei, 2016). Noble metal and alloy nanomaterials are very popular in biosensor application for their high-catalytic and biocompatible properties (Zhang et al., 2016). Moreover, research has indicated that bimetallic nanocrystals (NCs) with core-shell structures have higher stability and catalytic activity (Liu et al., 2016). Another example is the application of a microporous structure. Nanomaterials with a microporous structure have a large effective area and can exhibit capacitive properties. Ren et al. studied electrochemiluminescence (ECL)modified electrodes based on nanoporous cobalt (NPCo) with a three-dimensional network nanostructure, obtaining satisfactory catalytic activity and high structure stability (Lv et al., 2015).

On the other hand, there are increasing applications of nanocomposites in biosensor interface design. Azureen et al. studied gold-microrods (AuMRs), Pd-nanoparticles (PdNPs), and polyaniline (PANI) nanocomposite interfaces as peroxidase-mimic to form a label-free electrochemical biosensor (Mohamad et al., 2020). Xinmeng et al. combined two-dimensional hexagonal $\mathrm{NiCo}_{2} \mathrm{O}_{4}$ nanoplates with poly 3 , 4-ethylenedioxythiophene (PEDOT) and graphene to form nanocomposite for the detection of $\mathrm{H}_{2} \mathrm{O}_{2}$. PEDOT enhanced the stability of $\mathrm{NiCo}_{2} \mathrm{O}_{4} @ P E D O T / R G O$ interfaces as the binder existing between RGO and $\mathrm{NiCo}_{2} \mathrm{O}_{4}$ (Zhang et al., 2020). It was proved that the combination of nanomaterials and polymers was an effective solution for biosensor interface stability.

\section{Carbon-Based Nanomaterials}

Carbon-based materials have high electrocatalytic activity and electrical conductivity because of its special characteristics such as large surface area and numerous edge-plane-like defects (Inagaki et al., 2016). The good properties and various forms such as carbon flakes, carbon nanotubes, carbon nanofibers, and nanoporous carbons make carbon-based materials one of the significant materials in the application of biosensor interfaces. Among the carbon-based materials, grapheme- and graphenebased nanomaterials are the most common used materials owing to their high specific surface which can provide a platform for biomolecule loading and high conductivity that accelerates the electron transfer between the biomolecules and the surface (Fang et al., 2017). Zhou et al. proposed a chemiluminescent immunosensor based on graphene oxide (GO)-chitosan (CS) complex for the detection of $\mathrm{Hg}^{2+}$ (Zhou et al., 2019). GO can be used to immobilize antigens effectively in the biosensor because of its good water solubility and biocompatibility. CS has great biodegradability, film-forming ability, and biocompatibility. Thus, GO-CS composite can provide a stable environment for the fixation of biological receptors. The relative standard deviation (RSD) results of $0.01,1,100$, and $300 \mathrm{ng} \mathrm{ml}^{-1}$ $\mathrm{Hg}^{2+}$ detection ranged from $0.21 \%$ to $1.95 \%(n=5)$, which showed good stability of the biosensor (Zhou et al., 2019).

Moreover, the interface structures based on carbon-mixed materials have aroused a lot of research interest recently. Although carbon-based materials have great inherent properties, adding heteroatoms such as nitrogen, sulfur, phosphorus, and boron into the graphite carbon network can improve their performance further such as electrocatalytic activity, hydrophobicity, resistivity, charge transfer ability, and $\mathrm{pH}$ of the surface characteristics (Xiao et al., 2005; Ismagilov et al., 2009). Emran et al. reported a constructed electrode based on sulfur-doped microporous carbon (S-MC) for the selective detection of monobioactive molecules in the biological fluids and molecules secreted from living cells. The experiment results showed satisfactory sensing properties (Emran et al., 2018).

In addition to the materials mentioned above, there are many other types of nanomaterials. A list of nanomaterials commonly used in biosensor interfaces is shown in Table $\mathbf{1}$.

\section{Polymer}

Polymers are important materials in the construction of biosensor interfaces, because they are easy to process and their chemical and physical properties can be tailored as required. In particular, some polymers can be inert in an environment containing analytes, which is beneficial to the accuracy of detection. A survey of the literature revealed that polymers had gained a major position in many sensor devices. There are two main functions of polymers in biosensors. Conductive polymers are used as coating or encapsulating materials on the electrode surface, and nonconductive polymers are used to immobilize specific receptors on the sensor device (Adhikari and Majumdar, 2004; Cichosz et al., 2018). Nowadays, various polymers have been used in biosensors such as chitosan, agarose, polyethylene glycol, hydrogel, and polyelectrolyte (Sackmann, 1996). We will introduce three common types as examples here.

\section{Chitosan}

Chitosan has played a vital role in the development of biosensors due to its good biocompatibility, adhesion, and excellent filmforming ability, which means strong binding force for proteins and enzymes. Jain et al. prepared a biosensor interface by coimmobilizing the AChe and choline oxidase (ChOx) onto 
TABLE 1 | Nanomaterials used in biosensor interfaces.

\begin{tabular}{|c|c|c|c|}
\hline Nanomaterials & Properties & Applications & References \\
\hline $\begin{array}{l}\text { Noble metal nanoparticles } \\
\text { (Au, Ag) }\end{array}$ & $\begin{array}{l}\text { Localized surface plasmon resonance (LSPR), good } \\
\text { biocompatibility }\end{array}$ & Biomolecular recognition & Shen et al. (2012) \\
\hline $\begin{array}{l}\text { Noble metal nanoparticles } \\
(\mathrm{Pt}, \mathrm{Pd})\end{array}$ & High surface energy, good catalytic performance & $\begin{array}{l}\text { Nonenzymatic glucose oxidation, detection } \\
\text { signal amplification }\end{array}$ & Song et al. (2010) \\
\hline $\begin{array}{l}\text { Noble metallic } \\
\text { nanoclusters (NMNCs) }\end{array}$ & Good optical stability and biocompatibility & $\begin{array}{l}\text { Detection of tumor cells, being used as receptors } \\
\text { after biological modification }\end{array}$ & Tanaka et al. (2011) \\
\hline Quantum dots (QDs) & Quantum size effect, long fluorescence lifetime & Fluorescent biological probe & Wu et al. (2003) \\
\hline $\begin{array}{l}\text { Magnetic nanoparticles } \\
\text { (MNPs) }\end{array}$ & Magnetic property & High sensitivity sensor, immunoassay, catalyzing & $\begin{array}{l}\text { Omar and Abu-Reziq (2014), } \\
\text { Zhang et al. (2014) }\end{array}$ \\
\hline $\mathrm{SiO}_{2}$ & $\begin{array}{l}\text { High hardness, hydrophilic, good stability, easy to modify, } \\
\text { good biocompatibility, mesoporous feature }\end{array}$ & Controlled drug release system & Chen et al. (2012) \\
\hline $\mathrm{TiO}_{2}$ & $\begin{array}{l}\text { Nontoxic, stable chemical properties, mesoporous } \\
\text { properties, photocatalytic oxidation ability }\end{array}$ & $\begin{array}{l}\text { The target molecule can be loaded by the } \\
\text { channel inside the mesoporous structure }\end{array}$ & Rajh et al. (2014) \\
\hline Carbon nanotube & Good dispersity and stability & $\begin{array}{l}\text { Being used as receptors after biological } \\
\text { modification }\end{array}$ & Tsai et al. (2009) \\
\hline Graphene & $\begin{array}{l}\text { High thermal conductivity, large surface area, good } \\
\text { conductivity }\end{array}$ & Catalyst carrier, being receptors & Liu et al. (2008) \\
\hline Carbon dots (CDs) & Good luminescence performance, small size characteristic & Metal ion detection & Liu et al. (2012) \\
\hline
\end{tabular}

palladium nanoparticles (Pdnano). The Pdnano was adsorbed over molybdenum disulfide $\left(\mathrm{MoS}_{2}\right)$ nanostructures which were electrodeposited on the surface of gold electrode. The chitosan was used to improve interface stability and enzyme loading at the same time. By combining large surface area and stability of Pdnano, the biosensor showed good electrical performance (Jain et al., 2019). Ding et al. prepared a novel nanocomposite gel for cell immobilization and electrochemical studies by neutralizing chitosan nanocomposite formed from the reduction of tetrachloroauric acid in the original position of chitosan. The nanocomposite gel performed good immobilization ability and could maintain activity of immobilized living cells, which enhanced the biosensor stability greatly (Ding et al., 2007).

\section{Cellulose Membrane}

Cellulose, which is composed of glucose-based polymer chains, is the main component of plant cell walls and now is a widely used biopolymer (Kim et al., 2014). Cellulose has unique characteristics such as high transparency, good dimensional stability, and easy modification (El-Saied et al., 2020; Khattab et al., 2020). In addition, both cellulose and its derivatives exhibit high biocompatibility, which makes them suitable substrates for incorporation of biologically active substances. Since the typical carrier substrate for enzymes should be stable, inert, and resistant to mechanical changes, the cellulose matrix is also an ideal material for chemical covalent bonding, physical adsorption, and immobilization (Sternberg et al., 1988; Yun et al., 2008). Cellulose can be used to detect a variety of biomolecules, such as urea, lactic acid, glucose, genes, amino acid, cholesterol, and proteins.

In the study of Neelam et al., DAO from Pisum sativum (Pea) seedlings was extracted and purified by dialysis and gel filtration. Then, the DAO was covalently fixed on the surface of nitrocellulose membrane with glutaraldehyde. The obtained biological affinity supports demonstrated the high yield of immobilized DAO from pea seedlings and the enhanced storage stability of immobilized enzyme compared with free enzyme (Neelam et al., 2020), which indicated good stability of the cellulose membrane.

\section{Conducting Polymer}

Many polymers used in biosensors are conducting polymers. They can produce measurable signals by changing their electrical properties through the effects of different stimuli. This phenomenon can be observed due to their molecular and macroscopic structure. Thus, sensors can take advantage of physical changes when a conducting polymer is exposed to different chemicals (Janata and Josowicz, 2003; Moon et al., 2018).

Ammam et al. reported a glutamate microbial sensor based on polypyrrole (PPy), multiwalled carbon nanotubes (MWCNT), and glutamate oxidase (GluOx). The first layer of the interface structure is polypyrrole (PPy), which was used as a selective permeable membrane on the platinum $(\mathrm{Pt})$ electrode for the rejection of interferences. Compared with nonconducting polymer, the rate of alternating current electrophoretic deposition (AC-EPD) was significantly enhanced by using PPy for its high electrical conductivity. According to experimental data, the response to interferences remained constant for 30 days, proving that the PPy layer was stable and did not deteriorate. Upon the PPy layer, a controlled layer of MWCNT was followed by asymmetrical AC-EPD. This layer served as a support for enzyme deposition and increased the effective surface area of the sensor. The third step of interface construction involved depositing glutamate oxidase (GluOx) on the previously deposited carbon nanotubes by AC-EPD. The last step was to apply a thin polyurethane (PU) outer membrane to prevent the dissolution of deposited MWCNT and enzymes, which provided stability of the sensor (Ammam and Fransaer, 2010). Figure 2 shows the schematic representation of the glutamate sensor preparation, step 1: electropolymerization of polypyrrole; step 


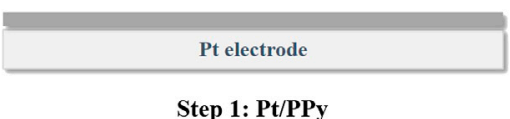

Step 1: Pt/PPy

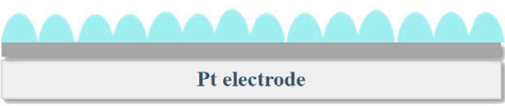

Step 2: Pt/PPy/MWCNT

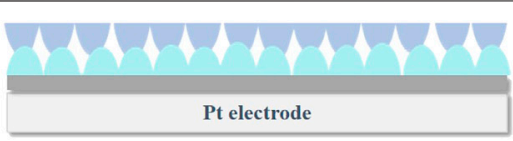

Step 3: Pt/PPy/MWCNT/GluOx

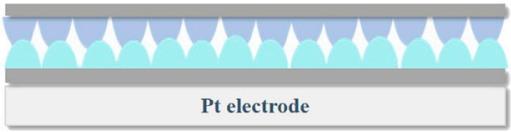

Step 4: Pt/PPy/MWCNT/GluOx/PU

FIGURE 2 | Schematic representation of the glutamate sensor preparation.

2: AC-EPD of MWCNT; step 3: AC-EPD of GluOx; step 4: spray coating with polyurethane outer layer (Ammam and Fransaer, 2010).

\section{Metal-Organic Framework}

In recent years, metal-organic framework (MOF), a new type of porous hybrid material, has attracted extensive interest in research. It contains metal ions and organic ligands, which are linked together by strong coordination bonds (Lee et al., 2009; Falcaro et al., 2014). Compared with inorganic nanomaterials, MOFs have potential advantages in biological applications due to their inherent biodegradability and the ability to use biocompatible components. Many types of MOFs have been applied to detect DNA, RNA, enzymes, small molecules, and so on. Others are applied in clinical diagnosis such as bioimaging (Blodgett, 1935).

Due to its superior properties of high surface area, large porosity, easy tenability of pore size, and modifiable surfaces (Lee et al., 2009; Falcaro et al., 2014), MOF is proved to be a suitable matrix for enzyme immobilization. Its large hierarchical surface area with remarkable porosity can provide high loading capacity, and its strong affinity can prevent enzyme leaching (Gkaniatsou et al., 2016).

Wang et al. immobilized xanthine oxidase (XOD) on a biocompatible copper fund organic skeleton nanofiber $(\mathrm{Cu}-$ MOF) membrane to develop a hypoxanthine and xanthine electrochemical biosensor. The $\mathrm{Cu}-\mathrm{MOF}$ was proved to have good topochemical properties, chemical stability, and thermal stability, which provided high storage stability of 20 days for the biosensor (Wang et al., 2019). Figure 3 shows the immobilization of XOD onto a copper-based metal organic framework fiber film and the fabrication of a XOD-electrochemical biosensor (Wang et al., 2019).

S. H. et al. reported a new method to enhance the lipase activity under low intensity ultrasound treatment by entrapping lipase within MOF in highly active conformation with the biomineralization method at room temperature. To evaluate the storage stability of different lipase forms, free lipase, sonicated lipase, and lipase-MOF were incubated at room temperature in phosphate buffer $(100 \mathrm{mM}, \mathrm{pH} 7.0)$ separately for 25 days. It was found that the residual activity of lipase-MOF, free lipase, and ultrasonic treatment lipase was 90, 68, and 66\%, respectively. Furthermore, the thermal stability of lipase-MOF was evaluated based on half-life in the range of $55-75^{\circ} \mathrm{C}$, which showed a 3.2-fold increment compared with the free lipase. The results indicated that the lipase-embedded MOF hybrid composites had high chemical and structural stability (GyepiGarbrah and Silerova, 2002).

\section{STRUCTURES AND PREPARATION METHODS OF STABLE BIOSENSOR INTERFACES}

\section{Monolayer Membrane Techniques}

Studies on monolayer membranes have mainly focused on three different preparation methods: the Langmuir-Blodgett (LB) technique, self-assembly monolayer (SAM) technique, and layer-by-layer (LBL) self-assembly technique. These techniques can be used to form an almost complete monolayer with a highly dense molecular ordered structure on the biofunctionalized surface. The loading of biomolecules on the surface is quite high, which can be beneficial for the development of highly specific and sensitive sensors. Careful tailoring of sensor surfaces by $\mathrm{LB} / \mathrm{SAM} / \mathrm{LBL}$ offers promising possibilities for development of new biosensor surfaces (Matharu et al., 2012). We will introduce LB and SAM in detail, which are the most widely used fabrication techniques in biosensor interfaces.

\section{Self-Assembled Monolayer Technique}

Several research groups have come up with different definitions of self-assembly. Maybe the best one is as follows: "self-assembly refers to the spontaneous formation of organized structures through a stochastic process that involves pre-existing components, is reversible, and can be controlled by appropriate design of the components, the environment, and the driving force" (Hosokawa et al., 1996; Campbell et al., 2002; Sahu et al., 2005).

The self-assembled monolayer (SAM) is formed by the chemical adsorption of molecules at the liquid-solid interface. 


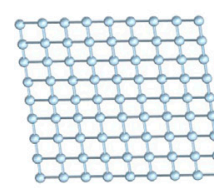

Cu-Based MOF Nanofiber

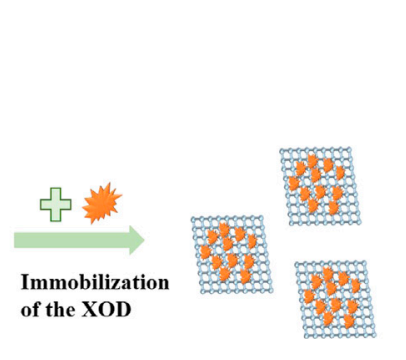

XOD@Cu-MOF

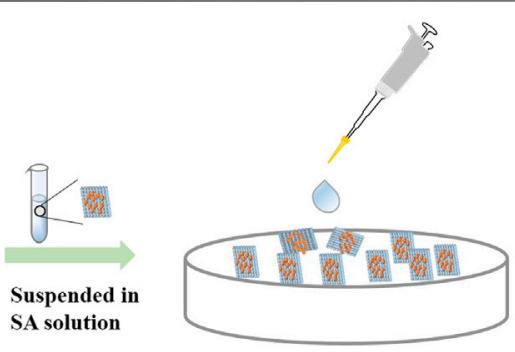

XOD-Electrochemical Biosensor

Xanthine oxidase(XOD)

SA buffer solution

glassy carbon electrode(GCA)

FIGURE 3 | The fabrication of XOD-electrochemical biosensor.

The general preparation method is to simply immerse a suitable solid support into a solution containing self-assembling molecules. During SAM growth, adsorption precedes molecular organization, which is different from the LB films (Langmuir, 1920).

There are three basic units of SAM: head groups bound to the surface of electrode, tail groups exposing at the SAM surface for chemical and physical properties adjustment, and spacer chains which are between head groups and tail groups for separation (Dubey et al., 2010).

Luo et al. designed a biosensor based on the SAM technique and alternating current (AC) electric capacitive sensing method. The biosensor interface was fabricated as the following steps: first, SAMs are formed using mercaptan containing alkyl chains fixed to the surface of the gold electrode via Au-S bonds by selfassembly techniques. Then, N-hydroxysuccinimide (NHS) and 1(3 dimethylaminopropyl)-3-ethyl carbon diimide hydrochloride (EDC) were used to form the amide bonds between the carboxyl group of the self-assembled monolayer and the amino group of the BPA antibodies (Luo et al., 2020). In this way, the interface between electrode and receptors (antibodies) had been built up. The next step was to fix the antibodies onto the interface, and then antibodies and antigens would have specific binding in the sample solution. After special treatment, the chip could be reused. The working principle diagram of the SAM-based biosensor is shown in Figure 4 (Luo et al., 2020).

\section{Langmuir-Blodgett Technique}

A variant of self-assembly is the assembly occurring at the gas-liquid interface. The Langmuir monolayer (LM) is a monolayer formed in this way. The LM can be transferred to a solid substrate to form a Langmuir-Blodgett (LB) membrane. In LB technique, molecular organization precedes adsorption (Langmuir, 1920). The earliest studies of monolayer membranes at the gas-liquid interface were proposed by Irving Langmuir in the late 1910s and early 1920s, and later he reported the transfer of fatty acid molecules from the water surface to solid carriers. The detailed transfer work was described by his student Katherine Blodgett. So, in honor of their outstanding contributions in this field, the monolayer membranes are called L-B membranes (Langmuir, 1920; Blodgett, 1935; Petty, 1983). A review reported by Matharu et al. introduced LM preparation, its characteristics at the gas-liquid interface, and the specific process of LB film deposition in detail. According to the review, nanomaterials and polymers are commonly used materials to prepare LB films (Matharu et al., 2012).

\section{Three-Dimensional Structure}

Most of the biosensor interfaces are two-dimensional (2D) arrays. However, biological molecules may require a more stable support environment for reaction in some situations. Compared with 2D structures, 3D structures could provide better structural stability. Moreover, considering that the real living environment of cells is in a complex $3 \mathrm{D}$ microenvironment, the $3 \mathrm{D}$ interface structure can better simulate the real cell environment in cell biosensors.

Wang et al. proposed a new 3D interface of biosensors. The preparation process is shown as follows: first, magnetic manganese phosphate nanoparticles $\mathrm{Fe}_{3} \mathrm{O}_{4}-\mathrm{Mn}_{3}\left(\mathrm{PO}_{4}\right)_{2} \mathrm{NPs}$ were synthesized by surface self-assembly monolayer (SAM) technology. Then, $\mathrm{Fe}_{3} \mathrm{O}_{4}-\mathrm{Mn}_{3}\left(\mathrm{PO}_{4}\right)_{2} \mathrm{NPs}$ were directly and firmly anchored on 3D nickel foam based on mussel-inspired adhesion technology. Finally, the 3D nickel foam was adsorbed on a magnetic electrode to construct the biosensor (Wang et al., 2018). As a matter of fact, real cells live in 3D space with a complex microenvironment (Liu et al., 2013). The 3D interface could provide a better simulated living environment for cells than $2 \mathrm{D}$ interfaces used mostly now. The method could be a new way for interface design of cell biosensors. However, although there are some good properties of 3D interfaces, the preparation of it is quite complex to operate. It will be a more practical method if some simplification can be made in preparation or other operation solutions can be found.

Peng et al. introduced tetrahedral DNA nanostructures (TDNs) into the formation of aptasensors. They combined aflatoxin B1 (AFB1) adaptors with TDNs and three- 


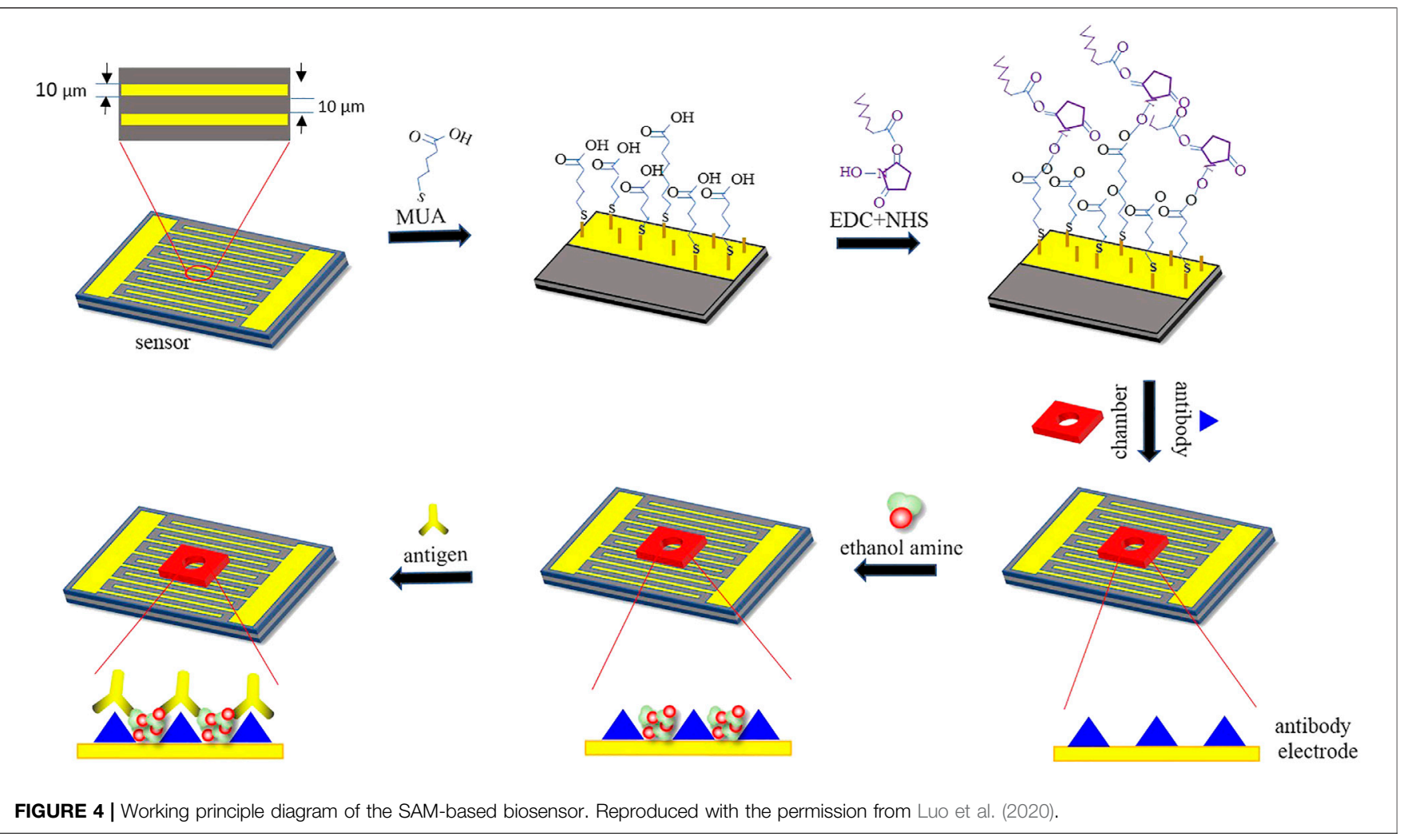

dimensionally ordered microporous $\mathrm{MoS}_{2}$-AuNPs hybrid (3DOM $\mathrm{MoS}_{2}$-AuNPs) thin film to construct the sensing interface (Peng et al., 2018). TDN is a new DNA structure with good mechanical rigidity, structural stability, precise controlled identification units, and specific orientation (Feng et al., 2017; Chen et al., 2014), which provides a stable environment for combination of biomolecules. Furthermore, the study showed that aptamers were expected to replace the traditional antigen-antibody binding reactions for its high specificity, good affinity, excellent stability, and easy preservation. The interface structure diagram of TDN-based aptasensor is shown in Figure 5 (Peng et al., 2018).

From the research examples in recent years, it is obvious that three-dimensional structure design presents new opportunities for interface stability of biosensors due to its special structure as well as the good properties owing to it. It may be a popular research direction in the future. However, on the other hand, there is still much space waiting to be studied in the field of $3 \mathrm{D}$ structure for its complex preparation process.

\section{Liquid Crystal Interfaces}

In addition to the above categories, there are many new methods and structures used in the biosensor interfaces. Here are several other examples proposed in the latest research studies.

Over the past 20 years, liquid crystal (LC)-based biosensors have been used for unlabeled sensing of biomolecules (Niu et al., 2017). Liquid crystal molecules can change their orientation due to the ability of adsorption or desorption at the interface and response to stimuli, which can be observed and reported by tracking the changes in liquid crystal optical birefringence in real time (Munir et al., 2016). Sundas et al. realized an unlabeled and enzyme-free detection of glucose by using boronic acidcoupled poly(styrene-b-acrylic acid) at liquid crystal/aqueous interfaces. The liquid crystal-based glucose biosensor had good stability within 40 days, low production cost, and simple immobilization technology (Munir and Park, 2018).

\section{S-layer}

S-layer, which is defined as "two-dimensional array of proteinaceous subunits forming the surface layer on prokaryotic cells," is a kind of highly porous protein lattices (Sleytr et al., 2014). It is also considered as the simplest biological protein or glycoprotein membrane during biological evolution (Sleytr et al., 2007). S-layer protein coatings can immobilize various receptors or provide a necessary environment for the recombination of functional membrane proteins and membraneactive peptides by self-assembling an ultrathin, porous twodimensional protein lattice on many surfaces and interfaces (Schuster and Sleytr, 2014). S-layer protein has three common applications in biosensor construction: firstly, it is very suitable for immobilization of biological molecules; secondly, S-layer protein can be fused with bioreceptors for recrystallization; thirdly, S lattice can be used as an anchoring scaffold and/or ion library to generate lipid film platforms (Schuster, 2018). The SUM (S-layer ultrafiltration membrane)-supported phospholipid bilayer is a highly isolated structure with a life span of up to $17 \mathrm{~h}$. 


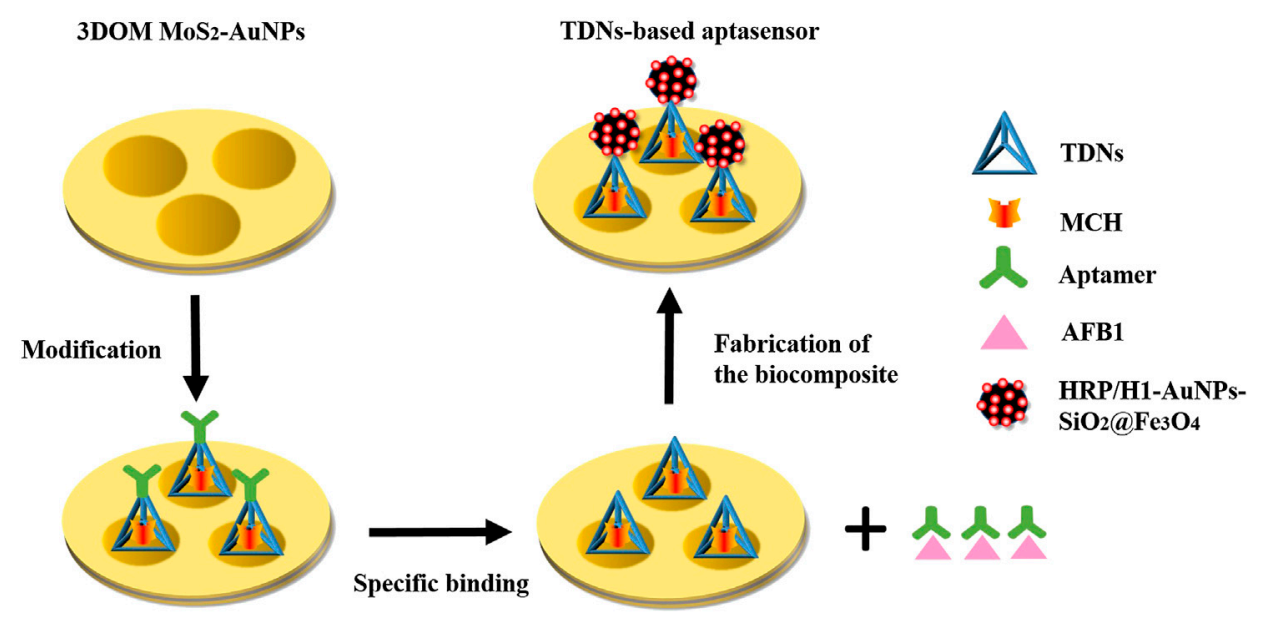

FIGURE 5 | Interface structure diagram of TDNs-based aptasensor.

However, by attaching an $\mathrm{S}$ lattice on both sides of the lipid membrane, the life span is significantly increased to about $24 \mathrm{~h}$ (Schuster et al., 2001; Gufler et al., 2004). Furthermore, if the lipid-head groups are cross-linked in contact with the domain on the S-layer protein directly, the life span of the composite membrane will further prolonged (Schuster and Sleytr, 2002). Therefore, S-layer protein is a promising method for preparation of stable and flowing lipid membranes on the biosensor interface.

Ferraz et al. reported a method for preparing a stable monolayer of cholesterol oxidase (ChOx) at the water-air interface. They prepared monomolecular films of ChOx and mixed films of $\mathrm{ChOx}$ and S-layer proteins which were extracted from B. sphaericus using a chaotropic agent (guanidine hydrochloride). Among these films, the mixed film containing S-layer showed more ideal performance than the pure ChOx film. S-layer proteins in the mixed film played an important role in protecting the active compound and stabilizing the molecular layers. Their study indicated that the use of S-layer proteins in biosensor production had a potential stabilizing effect (Ferraz et al., 2011).

\section{CONCLUSION}

This review illustrated the significant impact of interface stability on sensing performance through the analysis of biosensor structure and summarized some latest materials, structures, and preparation methods of biosensor interfaces by combining with the related research studies in recent years. Obviously, many innovative interface structures showed excellent stability and good sensing performance. It can be seen that nanomaterials and polymers as well as their compounds are the most popular materials in interface designs due to their excellent surface effects. Among these materials, noble metal particles, especially gold particles, are ideal materials for biosensor interfaces because of their good electrical conductivity, catalytic performance, and high chemical stability. Furthermore, there has been increasing new development in preparation methods such as monolayer membranes techniques, construction of three-dimensional interfaces, and other techniques of biosensor interfaces.

Since it involves different transduction modes, different analyte species (such as proteins, small molecules, and cells), different ways of functionalizing the active regions of the core sensors, and different application situations, it is difficult to compare the quality of various biosensor interfaces. In a word, the best way to obtain the right biosensor interface for a particular application is to assay the same biological model with various types of biosensing systems and choose the satisfactory one by comparing data.

Undoubtedly, much larger and faster development is observed in the case of studies on biosensor interface stability. However, there are still some challenges. Firstly, due to the specific selection of biosensors, a new method may only be applicable to a certain type or even a certain sensor but cannot be popularized. So, apart from selecting the right materials, researchers should also refine them and continually learn the mechanisms of reaction and mechanisms that allow an optimal biosensor interface to be obtained. Furthermore, the progress of interface science is limited by the development of material science and micronano technology to some extent. Searching for smart or new materials and techniques for biosensor production will be always on the way. In addition, although there has been good performance of many interface designs, they can only be completed in the laboratory because some of them need complicated preparation process and professional operations, which makes it difficult to put into extensive applications. Therefore, there is still a big room of biosensor interfaces for exploration.

However, the structures, materials, and interface preparation methods discussed above are far from all the categories because of 
the diversity and specificity of biological molecules detected, and the richness of interface techniques and materials. The purpose of this review is to do a simple summary of some commonly used materials and methods for biosensor interfaces so far and to provide part of new schemes of biosensor interfaces for reference. A more comprehensive and more detailed review about the stability solutions of biosensor interfaces needs further longterm and in-depth research. Perhaps, it will be the future research direction for us.

\section{AUTHOR CONTRIBUTIONS}

MS was responsible for methodology, formal analysis, investigation, data curation, and writing original draft. XL and

\section{REFERENCES}

Adhikari, B., and Majumdar, S. (2004). Polymers in sensor applications. Progress Polymer Sci. 29 (7), 699-766. doi:10.1016/j.progpolymsci.2004.03.002

Ammam, M., and Fransaer, J. (2010). Highly sensitive and selective glutamate microbiosensor based on cast polyurethane/AC-electrophoresis deposited multiwalled carbon nanotubes and then glutamate oxidase/electrosynthesized polypyrrole/Pt electrode. Biosens. Bioelectron. 25 (7), 1597-1602. doi:10.1016/j. bios.2009.11.020

Blodgett, K. B. (1935). Films built by depositing successive monomolecular layers on a solid surface. J. Am. Chem. Soc. 57, 1007-1022. doi:10.1021/ja01309a011

Campbell, D. J., Freidinger, E. R., and HastingsandQuerns, J. M. M. K. (2002). Spontaneous assembly of soda straws. J. Chem. Educ. 79, 201-202. doi:10.1021/ ed079p201

Cavalcanti, A., Shirinzadeh, B., Zhang, M., and Kretly, L. C. (2008). Nanorobot hardware architecture for medical defense. Sensors (Basel) 8 (5), 2932-2958. doi:10.3390/s8052932

Chen, M., Huang, C., He, C., Zhu, W., and Xu, Y., and Lu, Y. (2012). A glucoseresponsive controlled release system using glucose oxidase-gated mesoporous silica nanocontainers. Chem. Commun. (Camb) 48 (76), 9522-9524. doi:10. 1039/c2cc34290a

Chen, X., Zhou, G., Song, P., Wang, J., Gao, J., Lu, J., et al. (2014). Ultrasensitive electrochemical detection of prostate-specific antigen by using antibodies anchored on a DNA nanostructural scaffold. Anal. Chem. 86 (15), 7337-7342. doi:10.1021/ac500054x

Cichosz, S., Anna, M., and Zaborski, M. (2018). Polymer-based sensors: A review. Polymer Testing 67, 342-348. doi:10.1016/j.polymertesting.2018. 03.024

Ding, L., Hao, C., Xue, Y., and Ju, H. (2007). A bio-inspired support of gold nanoparticles-chitosan nanocomposites gel for immobilization and electrochemical study of K562 leukemia cells. Biomacromolecules 8 (4), 1341-1346. doi:10.1021/bm061224y

Dubey, M., Weidner, T., Gamble, L. J., and Castner, D. G. (2010). Structure and order of phosphonic acid-based self-assembled monolayers on $\mathrm{Si}(100)$. Langmuir 26 (18), 14747-14754. doi:10.1021/la1021438

Emran, M. Y., Shenashen, M. A., Morita, H., and El-Safty, S. A. (2018). One-step selective screening of bioactive molecules in living cells using sulfur-doped microporous carbon. Biosens. Bioelectron. 109, 237-245. doi:10.1016/j.bios.2018.03.026

El-Saied, H., Mostafa, A. M., Hasanin, M. S., Mwafy, E. A., and Mohammed, A. A. (2020). Synthesis of antimicrobial cellulosic derivative and its catalytic activity. J. King Saud Univ. Sci. 32, 436-442. doi:10.1016/j.jksus.2018. 06.007

Fang, X., Liu, J., Wang, J., Zhao, H., Ren, H., and Li, Z. (2017). Dual signal amplification strategy of $\mathrm{Au}$ nanopaticles/ $\mathrm{ZnO}$ nanorods hybridized reduced graphene nanosheet and multienzyme functionalized Au@ZnO composites for ultrasensitive electrochemical detection of tumor biomarker. Biosens. Bioelectron. 97, 218-225. doi:10.1016/j.bios.2017.05.055
$\mathrm{XZ}$ carried out conceptualization, validation, writing review and editing, supervision, project administration, and funding acquisition. ZP, SX, LJ, and HL were responsible for resources and writing original draft.

\section{FUNDING}

This work was supported by the Fundamental Research Funds for the Central Universities (project no. 2019CDYGZD006), the Postgraduate Education and Teaching Reform Research Project of Chongqing University (project no.cquyjg18323), Venture \& Innovation Support Program for Chongqing Overseas Returnees (Project No. cx2018017), and the National Natural Science Foundation of China (project no. 6137 7001).

Falcaro, P., Ricco, R., Doherty, C. M., Liang, K., Hill, A. J., and Styles, M. J. (2014). MOF positioning technology and device fabrication. Chem. Soc. Rev. 43 (16), 5513-5560. doi:10.1039/c4cs00089g

Feng, Q. M., Zhou, Z., Li, M. X., Zhao, W., and Xu, J.-J., and Chen, H.-Y. (2017). DNA tetrahedral scaffolds-based platform for the construction of electrochemiluminescence biosensor. Biosens. Bioelectron. 90, 251-257. doi:10.1016/j.bios.2016.11.060

Ferraz, H. C., Guimarães, J. A., Alves, T. L. M., and Constantino, C. J. L. (2011). Monomolecular films of cholesterol oxidase and S-Layer proteins. Appl. Surf. Sci. 257, 6535-6539. doi:10.1016/j.apsusc.2011.01.143

Gkaniatsou, E., Sicard, C., Ricoux, R., and Mahy, J.-P., Steunou, N., and Serre, C. (2016). Metal-organic frameworks: a novel host platform for enzymatic catalysis and detection. Materials Horizons 4 (1), 55-63. doi:10.1039. C6MH00312E

Gufler, P. C., Pum, D., Sleytr, U. B.., and Schuster, B. (2004). Highly robust lipid membranes on crystalline S-layer supports investigated by electrochemical impedance spectroscopy. Biochim. Biophys. Acta 1661 (2), 154-165. doi:10. 1016/j.bbamem.2003.12.009

Gyepi-Garbrah, S. H., and Silerova, R. (2002). The first direct comparison of selfassembly and langmuir-blodgett deposition techniques: two routes to highly organized monolayers. Phys. Chem. Chem. Phys. 4, (14) 3436-3442. doi:10. 1039/b201698b

Hernández, L. A., Del Valle, M. A., and Armijo, F. (2016). Electrosynthesis and characterization of nanostructured polyquinone for use in detection and quantification of naturally occurring dsDNA. Biosens. Bioelectron. 79, 280-287. doi:10.1016/j.bios.2015.12.041

Hosokawa, K., Shimoyama, I., and Miura, H. (1996). Two-dimensional micro-selfassembly using the surface tension of water. Sens. Actuators A. Phys. 57, $117-125$.

Inagaki, M., Toyoda, M., Soneda, Y., Tsujimura, S., and Morishita, T. (2016). Templated mesoporous carbons: synthesis and applications. Carbon 107, 448-473. doi:10.1016/j.carbon.2016.06.003

Isaac, A. M., Andrade, C. A. S., Balbino, V. Q., and Celsode Melo, P. (2017). Use of magnetically disentangled thiolated carbon nanotubes as a label-free impedimetric genosensor for detecting canine leishmania spp. infection. Carbon 117, 33-40. doi:10.1016/j.carbon.2017.02.031

Ismagilov, Z. R., Shalagina, A. E., Podyacheva, O. Y., Ischenko, A. V., Kibis, L. S., Boronin, A. I., et al. (2009). Structure and electrical conductivity of nitrogendoped carbon nanofibers. Carbon 47 (8), 1922-1929. doi:10.1016/j.carbon. 2009.02.034

Jain, U., Khanuja, M., Gupta, S., Harikumar, A., and Chauhan, N. (2019). Pd nanoparticles and molybdenum disulfide (MoS2) integrated sensing platform for the detection of neuromodulator. Process Biochem. 81, 48-56. doi:10.1016/j. procbio.2019.03.019

Janata, J., and Josowicz, M. (2003). Conducting polymers in electronic chemical sensors. Nat. Mater. 2, 19-24. doi:10.1038/nmat768

Moon, J.-M., Thapliyal, N., Hussain, K. K., and Goyal, R. N., and Shim, Y.-B. (2018). Conducting polymer-based electrochemical biosensors for 
neurotransmitters: a review. Biosensors Bioelectronics. 102, 540-552. doi:10. 1016/j.bios.2017.11.069

Khattab, T. A., Mohamed, A. L., and Hassabo, A. G. (2020). Development of durable superhydrophobic cotton fabrics coated with silicone/stearic acid using different cross-linkers. Mater. Chem. Phys. 249, 122981. doi:10.1016/j. matchemphys.2020.122981

Kim, J. H., Mun, S., Ko, H. U., Yun, G. Y., and Kim, J. (2014). Disposable chemical sensors and biosensors made on cellulose paper. Nanotechnology 25, 092001. doi:10.1088/0957-4484/25/9/092001

Kowalczyk, A. (2020). Trends and perspectives in DNA biosensors as diagnostic devices. Curr. Opinion Electrochemistry 23, 1-184. doi:10.1016/j.coelec.2020. 03.003

Langmuir, I. (1920). The mechanism of the surface phenomena of flotation. Trans. Faraday Soc. 15, 62-74

Lee, J., Farha, O. K., Roberts, J., Scheidt, K. A., and Nguyen, S. T., and Hupp, J. T. (2009). Metal-organic framework materials as catalysts. Chem. Soc. Rev. 38 (5), 1450-1459. doi:10.1039/b807080f

Liu, J. M, Lin, L. P, Wang, X. X, Lin, S.-Q., Cai, W.-L., Zhang, L.-H., et al. (2012). Highly selective and sensitive detection of $\mathrm{Cu} 2+$ with lysine enhancing bovine serum albumin modified-carbon dots fluorescent probe. Analyst 137 (11), 2637-2642. doi:10.1039/c2an35130g

Liu, Z., Robinson, J. T., Sun, X., and Dai, H. (2008). PEGylated nanographene oxide for delivery of water-insoluble cancer drugs. J. Am. Chem. Soc. 130 (33), 10876-10877. doi:10.1021/ja803688x

Liu, X., Chen, L., Liu, H., Yang, G., Zhang, P., Han, D., et al. (2013). Bio-inspired soft polystyrene nanotube substrate for rapid and highly efficient breast cancercell capture. NPG Asia Mater 5 (9), e63. doi:10.1038/am.2013.43

Liu, Y., Ma, H., Gao, J., Wu, D., Ren, X., Yan, T., Pang, X., and Wei, Q. (2016). Ultrasensitive electrochemical immunosensor for SCCA detection based on ternary $\mathrm{Pt} / \mathrm{PdCu}$ nanocube anchored on three-dimensional graphene framework for signal amplification. Biosens. Bioelectron. 79, 71. doi:10.1016/ j.bios.2015.12.013

Luo, H., Lin, X., Peng, Z., and Song, M., and Jin, L. (2020). Rapid and sensitive detection of bisphenol A based on self-assembly. Micromachines 11 (1), 41. doi:10.3390/mi11010041

Lv, X., Li, Y., Yan, T., and Pang, X., Cao, W., Du, B., et al. (2015). Electrochemiluminescence modified electrodes based on RuSi@Ru(bpy)3(2+) loaded with gold functioned nanoporous $\mathrm{CO} / \mathrm{Co} 3 \mathrm{O} 4$ for detection of mycotoxin deoxynivalenol. Biosens. Bioelectron. 70, 28-33. doi:10.1016/j. bios.2015.03.020

Matharu, Z., Bandodkar, A. J., Gupta, V., and Malhotra, B. D. (2012). Fundamentals and application of ordered molecular assemblies to affinity biosensing. Chem. Soc. Rev. 41 (3), 1363-1402. doi:10.1039/c1cs15145b

Matharu, Z., Daggumati, P., Wang, L., Dorofeeva, T. S., Li, Z., and Seker, E. (2017). Nanoporous-gold-based electrode morphology libraries for investigating structure-property relationships in nucleic acid based electrochemical biosensors. ACS Appl. Mater. Interfaces 9 (15), 12959-12966. doi:10.1021/ acsami.6b15212

Mohamad, A., Rizwan, M., Keasberry, N. A., Nguyen, A. S., and Lam, T. D., and Ahmed, M. U. (2020). Gold-microrods/Pd-nanoparticles/polyanilinenanocomposite-interface as a peroxidase-mimic for sensitive detection of tropomyosin. Biosens. Bioelectron. 155, 112108. doi:10.1016/j.bios.2020.112108

Munir, S., and Park, S. Y. (2018). Label- and enzyme-free detection of glucose by boronic acid-coupled poly(styrene-b-acrylic acid) at liquid crystal/aqueous interfaces. Anal. Chim. Acta 1032, 122-129. doi:10.1016/j.aca.2018.05.039

Munir, S., Kang, I., and Park, S. (2016). Polyelectrolytes functionalized nematic liquid crystal-based biosensors: an overview. Trac. Trends Anal. Chem. 83, 80-94. doi:10.1016/j.trac.2016.08.007

Neelam, V., Lovely, S., Anjum, G., Hooda, V., and Hooda, V. (2020). Novel approach using activated cellulose film for efficient immobilization of purified diamine oxidase to enhance enzyme performance and stability. Prep. Biochem. Biotechnol. 50 (5), 468-476. doi:10.1080/10826068.2019.1709976

Nicu, L., and Leichle, T. (2008). Biosensors and tools for surface functionalization from the macro-to the nanoscale: the way forward. J. Applied Phys. 104 (11), 111101. doi:10.1063/1.2973147

Niu, X., Zhong, Y., Chen, R., Wang, F., and Luo, D. (2017). Highly sensitive and selective liquid crystal optical sensor for detection of ammonia. Opt. Express 25 (12), 13549. doi:10.1364/OE.25.013549
Omar, S., and Abu-Reziq, R. (2014). Palladium nanoparticles subborted on magnetic organic-silica hybrid nanoparticles. J. Phys. Chem. C 118 (51), 30045-30056. doi:10.1021/jp510472t

Parlak, O., İncel, A., Uzun, L., and Turner, A. P. F., and Tiwari, A. (2017). Structuring $\mathrm{Au}$ nanoparticles on two-dimensional $\mathrm{MoS}_{2}$ nanosheets for electrochemical glucose biosensors. Biosens. Bioelectron. 89, 545-550. doi:10. 1016/j.bios.2016.03.024

Peng, G., Li, X., Cui, F., Qiu, Q., Chen, X., and Huang, H. (2018). Aflatoxin B1 electrochemical aptasensor based on tetrahedral DNA nanostructures functionalized three dimensionally ordered macroporous MoS2-AuNPs film. ACS Appl. Mater. Interfaces 10 (21), 17551-17559. doi:10.1021/acsami.8b01693

Petty, M. C. (1983). Langmuir-blodgett films. Endeavour, 7 (2), 65-69. doi:10.1016/ S0160-9327(83)80004-0

Qian, J., Zhu, W., Li, M., Xu, X., Yu, J., Cui, D., et al. (2014). Nanohybrids of quantum dots and cytochrome P450 for light-driven drug metabolism. J. Electroanal. Chem. 733, 27-32. doi:10.1016/j.jelechem.2014.09.012

Rajh, T., Dimitrijevic, N. M., Bissonnette, M., and Koritarov, T., and Konda, V. (2014). Titanium dioxide in the service of the biomedical revolution. Chem. Rev. 114 (19), 10177-10216. doi:10.1021/cr500029g

Sackmann, E (1996). Supported membranes: scientific and practical applications. Science 271 (5245), 43-48.

Sahu, S., Yin, P., and Reif, J. H. (2005). “A self-assembly model of time-dependent glue strength," in International Workshop on DNA-Based Computers, (Berlin, Heidelberg: Springer).

Schmidt, J. J., and Montemagno, C. D. (2004). Bionanomechanical systems. Annu. Rev. Mater. Res. 34 (1), 315-337. doi:10.1146/annurev.matsci.34.040203.115827

Schuster, B., Pum, D., Sára, M., Braha, O., and Bayley, H., and Sleytr, U. B. (2001). S-layer ultrafiltration membranes: a new support for stabilizing functionalized lipid membranes. Langmuir 17 (2), 499-503. doi:10.1021/la0008784

Schuster, B., and Sleytr, U. B. (2014). Biomimetic interfaces based on S-layer proteins, lipid membranes and functional biomolecules. J. R. Soc. Interface 11 (96), 20140232. doi:10.1098/rsif.2014.0232

Schuster, B., and Sleytr, U. B. (2002). The effect of hydrostatic pressure on S-layersupported lipid membranes. Biochim. Biophys. Acta Biomembr. 1563 (1), 29-34. doi:10.1016/s0005-2736(02)00370-x

Schuster, B. (2018). S-layer protein-based biosensors. Biosensors 8 (2), 40. doi:10. 3390/bios 8020040

Shen, W., Deng, H. M., and Gao, Z. Q. (2012). Gold nanoparticle-enabled real-time ligation chain reaction for ultrasensitive detection of DNA. J. Am. Chem. Soc. 134 (36), 14678-14681. doi:10.1021/ja306265n

Shi, W. T., and Ma, Z. F. (2011). A novel label-free amperometric immunosensor for carcinoembryonic antigen based on redox membrane. Biosens. Bioelectron. 26 (6), 3068-3071. doi:10.1016/j.bios.2010.11.048

Sleytr, U. B., Schuster, B., Eva-Maria, E., and Dietmar, P. (2014). S-layers: principles and applications. FEMS Microbiol. Rev. 38 (5), 823-864. doi:10.1111/1574-6976.12063

Sleytr, U. B., Huber, C., Ilk, N., Pum, D., Schuster, B., and Egelseer, E. M. (2007). S-layers as a tool kit for nanobiotechnological applications. FEMS Microbiol. Lett. 267 (2), 131-144. doi:10.1111/j.1574-6968.2006.00573.x

Song, Z. J., Yuan, R., Chai, Y. Q., Zhuo, Y., Jiang, W., Su, H., et al. (2010). Horseradish peroxidase-functionalized Pt hollow nanospheres and multiple redox probes as trace labels for a sensitive simultaneous multianalyte electrochemical immunoassay. Chem. Commun. (Camb) 46 (36), 6750-6752. doi:10. 1039/c0cc01537g

Sternberg, R., Bindra, D. S., Wilson, G. S., and Thevenot, D. R. (1988). Covalent enzyme coupling on cellulose acetate membranes for glucose sensor development. Anal. Chem. 60, 2781-2786. doi:10.1021/ac00175a028

Sun, X., and Ma, Z. (2012). Highly stable electrochemical immunosensor for carcinoembryonic antigen. Biosens. Bioelectron. 35 (1), 470-474. doi:10.1016/j. bios.2012.02.061

Tanaka, S.-I., Miyazaki, J., Tiwari, D. K, and Jin, T., and Inouye, Y. (2011). Fluorescent platinum nanoclusters: synthesis, purification, characterization, and application to bioimaging. Angew Chem. Int. Ed. Engl. 50 (2), 431-435. doi:10.1002/anie. 201004907

Thévenot, D. R., Toth, K., Durst, R. A., and Wilson, G. S. (2001). Electrochemical biosensors: recommended definitions and classification. Biosens. Bioelectron. 16 (1-2), 121-131. doi:10.1016/s0956-5663(01)00115-4

Thiagarajan, V., Madhurantakam, S., Sethuraman, S., and Rayappan, J. B. B., and Krishnan, U. M. (2016). Nano interfaced biosensor for detection of choline in 
triple negative breast cancer cells. J. Colloid Interface Sci. 462, 334-340. doi:10. 1016/j.jcis.2015.10.014

Tsai, T. W., Heckert, G., Neves, L. F., Tan, Y., Kao, D.-Y., Harrison, R. G., et al. (2009). Adsorption of glucose oxidase onto single-walled carbon nanotubes and its application in layer-by-layer biosensors. J. Anal. Chem. 81 (19), 7917-7925. doi:10.1021/ac900650r

Turner, A. P. F. (2015). Biosensors: fundamentals and applications - historic book now open access. Biosens. Bioelectron. 65, A1. doi:10.1016/j.bios.2014.10.027

Wang, Q., Zhou, Q., Zhang, Q., Shi, R., Ma, S., Zhao, W., and Zhou, M. (2018). Fabrication of novel superoxide anion biosensor based on 3D interface of mussel-inspired Fe 3 O 4 -Mn 3 (PO3) 2 @Ni foam. Talanta 179, 145-152. doi:10.1016/j.talanta.2017.10.054

Wang, Z., Ma, B., Shen, C., Lai, O.-M., Tan, C.-P., and Cheong, L.-Z. (2019). Electrochemical biosensing of chilled seafood freshness by xanthine oxidase immobilized on copper-based metal-organic framework nanofiber film. Food Analytical Methods 12 (8), 1715-1724. doi:10.1007/s12161-019-01513-8

Wu, X., Liu, H., Liuj, J., Haley, K. N., Treadway, J. A., Larson, J. P., et al. (2003). Immunofluorescent labeling of cancer marker Her2 and other cellular targets with semiconduetor quantum dots. Nat. Biotechnol. 21 (1), 452. doi:10.1038/ nbt0403-452b

Xiao, K., Liu, Y., Hu, P., and Yu, G., Sun, Y., and Zhu, D. (2005). n-Type field-effect transistors made of an individual nitrogen-doped multiwalled carbon nanotube. J. Am. Chem. Soc. 127 (24), 8614-8617. doi:10.1021/ja042554y

Yun, G. Y., Kim, H. S., Kim, J., Kim, K., and Yang, C. (2008). Effect of aligned cellulose film to the performance of electro-active paper actuator. Sens. Actuators A Phys. 141, 530-535. doi:10.1016/j.sna.2007.10.014

Zhang, X., Zhao, Y., Wu, Y., and Mao, Z. (2020). Two-dimensional hexagonal NiCo2O4 Nanoplates@PEDOT/RGO nanocomposite: a design and construction high selective $\mathrm{H} 2 \mathrm{O} 2$ sensing interface. J. Electrochem. Soc. 167 (6), 67519. doi:10.1149/1945-7111/ab82fd

Zhang, Y., Li, J., Wang, Z., Ma, H., Wu, D., Cheng, Q., et al. (2016). Label-free electrochemical immunosensor based on enhanced signal amplification between Au@Pd and CoFe2O4/graphene nanohybrid. Sci. Rep. 6 (1), 23391. doi:10.1038/srep23391

Zhang, Y. T., Li, D., Yu, M., Ma, W., and Guo, J., and Wang, C. (2014). Fe3O4/ PVIM-Ni2+ magnetic composite microspheres for highly specific separation of histidine-rich proteins. ACS Appl. Mater. Interfaces 6 (11), 8836-8844. doi:10. 1021/am501626t

Zhang, Y., and Wei, Q. (2016). The role of nanomaterials in electroanalytical biosensors: a mini review. J. Electroanal. Chem. 781, 401-409. doi:10.1016/j. jelechem.2016.09.011

Zhao, W., Ni, Y., Zhu, Q., Fu, R., Huang, X., and Shen, J. (2013). Innovative biocompatible nanospheres as biomimetic platform for electrochemical glucose biosensor. Biosens. Bioelectron. 44, 1-5. doi:10.1016/j.bios.2012.12.036

Zhou, X., Zhang, S., Shi, J., Zhao, K., and Deng, A., and Li, J. (2019). An ultrasensitive competitive chemiluminescence immunosensor coupled flow injection cell modified by oxidized graphene-chitosan for the detection of $\mathrm{Hg}^{2+}$. Microchem. J. 149, 103997. doi:10.1016/j.microc.2019.103997

Zhu, Z., Gao, F., Lei, J., Dong, H., and Ju, H. (2012). A competitive strategy coupled with endonuclease-assisted target recycling for DNA detection using silvernanoparticle-tagged carbon nanospheres as labels. Chem. Eur J. 18 (43), 13871-13876. doi:10.1002/chem.201201307

Conflict of Interest: The authors declare that the research was conducted in the absence of any commercial or financial relationships that could be construed as a potential conflict of interest.

Copyright (c) 2021 Song, Lin, Peng, Xu, Jin, Zheng and Luo. This is an open-access article distributed under the terms of the Creative Commons Attribution License (CC $B Y)$. The use, distribution or reproduction in other forums is permitted, provided the original author(s) and the copyright owner(s) are credited and that the original publication in this journal is cited, in accordance with accepted academic practice. No use, distribution or reproduction is permitted which does not comply with these terms. 